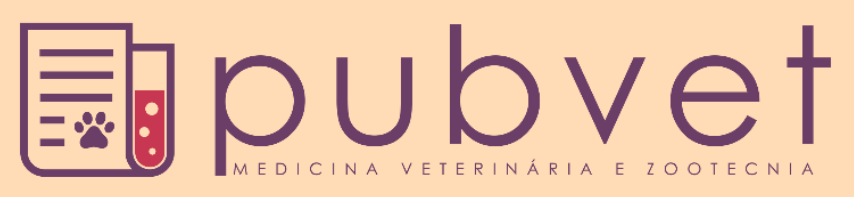

https://doi.org/10.31533/pubvet.v14n1a495.1-4

\title{
Uso da ozonioterapia como auxiliar no tratamento de cão portador de leishmaniose: relato de caso
}

\author{
Jéssica Oliveira Santos Gonçalves $^{1 \oplus}$, Priscila de Oliveira Paiva ${ }^{1 \oplus}$, Lyana Brasil Gomes de Oliveira ${ }^{2} \bullet$ \\ ${ }^{1}$ Estudante de graduação de Medicina Veterinária, Universidade Federal de Sergipe. \\ ${ }^{2}$ Médica veterinária autônoma, graduada pela Universidade Federal de Sergipe. \\ Autor para correspondência, E-mail: jessica.osg@live.com
}

\begin{abstract}
Resumo. A Leishmaniose Visceral Canina (LVC) é uma doença infecciosa de caráter zoonótico, transmitida por mosquito pertencente à família dos flebotomíneos. Os sinais clínicos frequentemente observados envolvem linfadenopatia, insuficiência renal, poliartrite, esplenomegalia, sinais cutâneos como hiperqueratose, alopecia, onicogrifose, entre outros. $\mathrm{O}$ tratamento possui custo elevado, e consiste na associação de drogas, que podem causar efeitos colaterais severos no paciente devido sua toxicidade. Buscando reduzir os efeitos colaterais, melhorar a imunidade e disposição dos pacientes, médicos veterinários tem buscado terapias auxiliares para minimizar os danos do tratamento, promovendo maior bem-estar ao paciente. Desta forma, este trabalho, busca relatar o caso de uma cadela diagnosticada clinicamente e sorologicamente com Leishmaniose, que obteve um resultado positivo na associação de aplicações do gás ozônio ao tratamento medicamentoso.
\end{abstract}

Palavras-chave: ozonioterapia, ozônio, leishmaniose, alternativas terapêuticas, medicina veterinária integrativa

\section{Use of ozoniotherapy as aid in Leishmaniosis dog treatment: case report}

\begin{abstract}
Canine Visceral Leishmaniosis (LVC) is a zoonotic infectious disease transmitted by a mosquito belonging to the family of phlebotomines. The clinical signs often observed involve lymphadenopathy, renal failure, polyarthritis, splenomegaly, skin signs such as hyperkeratosis, alopecia, onicogrifosis, among others. Treatment has high cost, and consists of the association of drugs, which can cause severe side effects on the patient due to its toxicity. Seeking to reduce side effects, improve patients' immunity and disposition, veterinarians have sought auxiliary therapies to minimize treatment damage, promoting greater well-being to the patient. Thus, this study seeks to report the case of a dog clinically and serologically diagnosed with Leishmaniosis, which obtained a positive result in the association of ozone gas applications with drug treatment.
\end{abstract}

Keywords: ozone therapy, ozone, leishmaniasis, therapeutic alternatives, integrative veterinary medicine

\section{Uso de la ozonioterapia como ayuda en el tratamiento leishmaniosis canina: reporte de un caso}

Resumen. La leishmaniosis visceral canina (LVC) es una enfermedad infecciosa zoonótica transmitida por un mosquito perteneciente a la familia de las flebotominas. Los signos clínicos a menudo observados involucran linfadenopatía, insuficiencia renal, poliartritis, esplenomegalia, signos cutáneos como hiperqueratosis, alopecia, onicogrifosis, entre otros. El tratamiento tiene un alto costo, y consiste en la asociación de fármacos, que pueden causar efectos secundarios graves en el paciente debido a su toxicidad. Buscando reducir 
los efectos secundarios, mejorar la inmunidad y disposición de los pacientes, los veterinarios han buscado terapias auxiliares para minimizar el daño al tratamiento, promoviendo un mayor bienestar para el paciente. Por lo tanto, este estudio busca informar el caso de un perro diagnosticado clínica y serológicamente con Leishmaniosis, que obtuvo un resultado positivo en la asociación de aplicaciones de gas de ozono con el tratamiento farmacológico.

Palabras clave: ozonioterapia, ozono, leishmaniosis, alternativas terapéuticas, medicina veterinaria integrativa

\section{Introdução}

A leishmaniose visceral canina (LVC) é uma patologia causada por um protozoário do gênero Leishmania, acometendo principalmente cães, roedores e canídeos silvestres (Costa, 2011). No Brasil, a transmissão geralmente ocorre pela picada do "mosquito palha" - Lutzomyia longipalpis. Por se tratar de um inseto muito pequeno e que se reproduz na matéria orgânica, o controle deste vetor torna-se difícil (Schimming \& Silva, 2012). Sousa \& Winck (2018) traz em seu trabalho, que a leishmania, ao entrar em contato com o hospedeiro, o sistema imunológico é ativado, gerando uma resposta imune efetiva para que os parasitos não se multipliquem. Quando isso não ocorre, estes se espalham para os órgãos via fagócitos mononucleares. Os animais podem apresentar anemia entre leve e moderada, aplasia medular, anemia não regenerativa, hiperproteinemia, trombocitopenia e leucocitose.

$\mathrm{O}$ ozônio $\mathrm{O}_{3}$ é um gás instável, incolor e de odor característico. Na natureza pode ser obtido através da recombinação das moléculas de oxigênio após uma descarga elétrica, ou por plantas e plânctons. Possui características germicidas e desinfetantes o que amplia a gama de sua utilização. Sua aplicação com fim medicinal iniciou-se por volta de 1890; porém, os estudos clínicos iniciaram em 1950 (Penido et al., 2010). Devido suas propriedades fungicidas, bactericida, viricida, oxidante, atuando ainda na oxigenação tecidual, reduzindo agregação plaquetária, estimulando o sistema imune via sistema retículo endotelial (Vilarindo et al., 2013).

Ao penetrar no organismo, o ozônio proporciona melhor oxigenação e metabolismo, pois a reatividade com os componentes sanguíneos é muito rápida (Silva et al., 2018). A administração pode ser feita por via subcutânea, intramuscular, intradiscal, intracavitária, intravaginal, intrauretral, auto-hemoterapia maior e menor (Bocci et al., 2011) e por via tópica, através de óleo ozonizado, água, e aplicação direta com utilização de sacos plásticos envoltos, técnica conhecida como "bag" (Silva et al., 2018).

\section{Descrição do caso}

Em 14 de novembro de 2018, foi realizado o atendimento domiciliar de uma fêmea canina, da raça labrador, castrada, idade de oito anos, $27 \mathrm{~kg}$. A principal queixa da tutora eram lesões escamosas em várias regiões do corpo (Figura 1a e 1b) que não cicatrizavam, crescimento demasiado das unhas e que o animal apresentava apatia. Desta forma, foi então coletado amostras sanguíneas e encaminhado para análise bioquímico e sorológica. Na tabela 1 são apresentados os resultados destes exames.

Tabela 1. Resultados dos exames sanguíneos coletados de fêmea canina portadora de leishmaniose

\begin{tabular}{|c|c|c|}
\hline Data & Exame & Resultados \\
\hline $14 / 11 / 2018$ & Hemograma & $\begin{array}{l}\text { Anemia microcítica normocrômica, neutrofilia relativa, linfopenia relativa e } \\
\text { absoluta, eosinopenia relativa e absoluta, trombocitopenia e hiperproteinemia. } \\
\text { eritrócitos: 5,5 milhões } / \mathrm{mm}^{3} \text {, Hemoglobina: } 10,1 \mathrm{~g} / \mathrm{dl} \text {, Hematócrito:30 \%, } \\
\text { V.C.M:54,55 } \mathrm{u}^{3} \text {, Proteína plasmática: } 10,40, \text { Leucócitos: } 8,90 \mathrm{mil} / \mathrm{mm}^{3} \text {, } \\
\text { Linfócitos: } 8,00 \% 712 / \mathrm{mm}^{3} \text {, Contagem plaquetária: } 168 \mathrm{mil} / \mathrm{mm}^{3}\end{array}$ \\
\hline \multirow{3}{*}{ 20/11/2018 } & \multirow{3}{*}{ Elisa } & Reagente \\
\hline & & CUT OFF: 0,214 \\
\hline & & Valor da OD*: 0,295 \\
\hline \multirow{2}{*}{ 20/11/2018 } & \multirow{2}{*}{ Imunofluorescência Indireta } & Reagente \\
\hline & & Diluição 1/640 \\
\hline
\end{tabular}


O protocolo adotado foi uma associação de Domperidona $10 \mathrm{mg}$, na dose de $30 \mathrm{mg}$ a cada 12 horas por 30 dias e Alopurinol $300 \mathrm{mg}$ via oral na dose de $450 \mathrm{mg}$ a cada 12 horas por 30 dias e Miltefosina a $2 \%$ na dose de $2,8 \mathrm{ml}$ a cada 24 horas por 28 dias mais aplicações de ozônio (Tabela 2).

Tabela 2. Protocolo de aplicações de ozônio em fêmea canina portadora de leishmaniose

\begin{tabular}{lc}
\hline Data & Protocolo \\
\hline $29 / 11$ & $60 \mathrm{ml}$ via retal na concentração de 20mcg e Auto-hemoterapia menor $2,5 \mathrm{ml}$ de sangue e de O3 \\
$04 / 12$ & Peri-lesional na concentração de $14 \mathrm{mcg}$ e retal na concentração de $20 \mathrm{mcg}$ \\
$06 / 12$ & Auto-hemoterapia menor $5 \mathrm{~mL}$ de sangue e $5 \mathrm{~mL}$ de O3 e $60 \mathrm{ml}$ retal na concentração de $20 \mathrm{mcg}$ \\
$11 / 12$ & Peri-lesional na concentração de $14 \mathrm{mcg}$ e soro ozonizado via subcutâneo \\
$13 / 12$ & Auto-hemoterapia menor $5 \mathrm{~mL}$ de sangue e $5 \mathrm{~mL}$ de O3 e $60 \mathrm{ml}$ retal na concentração de $20 \mathrm{mcg}$ \\
$18 / 12$ & Soro ozonizado subcutâneo e intra retal na concentração de 20mcg \\
$28 / 12$ & Auto-hemoterapia na concentração de 20mcg e $60 \mathrm{ml}$ intra retal \\
$04 / 01$ & Auto-hemoterapia na concentração de 20mcg e $60 \mathrm{ml}$ intra retal \\
$06 / 02$ & Auto-hemoterapia na concentração de 20mcg e $60 \mathrm{ml}$ intra retal \\
$28 / 02$ & Auto-hemoterapia na concentração de 20mcg e $60 \mathrm{ml}$ intra retal \\
$27 / 03$ & Auto-hemoterapia na concentração de 20mcg e $60 \mathrm{ml}$ intra retal \\
$19 / 04$ & Auto-hemoterapia na concentração de 20mcg e $60 \mathrm{ml}$ intra retal \\
\hline
\end{tabular}

A melhora na pele foi notória por volta da quarta aplicação do ozônio (Figura 1c e 1d). A tutora relatou que no dia posterior a aplicação, o animal demonstrava mais disposição para jogos e melhora no apetite. A paciente realizou todo o tratamento com o Milteforam apresentando um quadro diarreico por volta do vigésimo dia, permanecendo a sintomatologia por dois dias. Foi adicionado ao tratamento, Enterogermina Plus sendo administrada uma ampola via oral durante 10 dias. Ao fim do tratamento, os exames sorológicos e hematológicos foram repetidos, apresentando os resultados conforme a tabela 3.

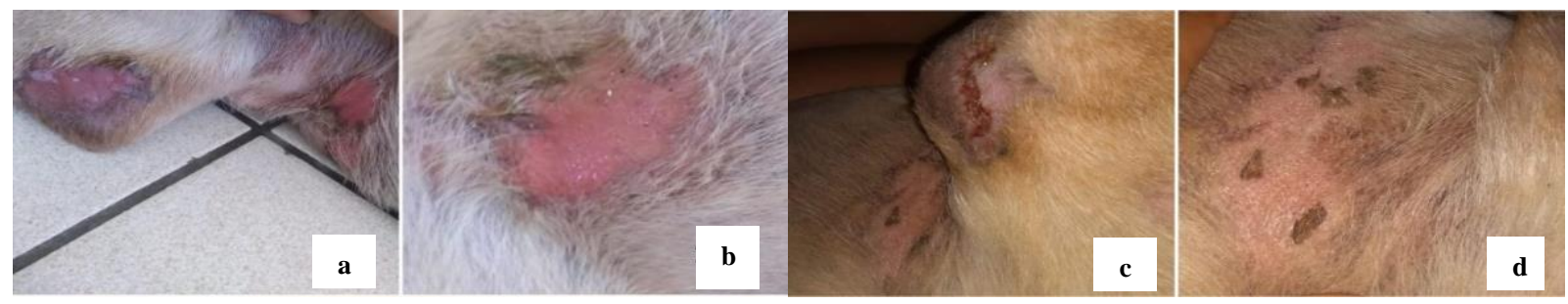

Figura 1. Lesão em região de cotovelo (a) e lesão em região axilar (b) no dia 28/11/2018. Lesão em região de cotovelo (c) e lesão em região axilar (d) no dia 4/12/2018. Nota-se a melhora na cicatrização.

Tabela 3. Resultados dos exames sanguíneos coletados de fêmea canina portadora de leishmaniose, após aplicações de ozônio

\begin{tabular}{lll}
\hline Data & Exame & Resultados \\
\hline & & Anemia Microcítica Hipocrômica; Hiperproteinemia \\
& & Eritrócitos: 5,4 milhões $/ \mathrm{mm}^{3}$ \\
$04 / 01 / 2019$ & Hemograma & Leucócitos: $8,60 \mathrm{mil} / \mathrm{mm}^{3}$ Linfócitos: $16,00 \%$ Monócitos: $3,00 \%$ \\
& & Contagem plaquetária: $300 \mathrm{mil} / \mathrm{mm}^{3}$ \\
\hline \multirow{2}{*}{$08 / 01 / 2019$} & ELISA & Indeterminado \\
& & Cut Off: 0,211 \\
\hline $08 / 01 / 2019$ & Valor Da Od*: 0,185 \\
\hline
\end{tabular}

\section{Discussão}

O protocolo medicamentoso de eleição foi à associação da Miltefosina com Alopurinol e Domperidona. O Alopurinol é uma droga leishmaniostática, que possui uma ação positiva quando combinada com outras medicações. A Domperidona, mesmo não exercendo atividade direta sobre as leishmanias, possui ação imunomoduladora e tem sido eficaz no controle e redução dos sinais clínicos 
de cães soropositivos para LVC (Araújo et al., 2018). Sousa \& Winck (2018) trazem em seu estudo que é necessário que avanços tecnológicos em relação ao tratamento de cães infectados, a fim de desenvolver medicamentos mais acessíveis, menos tóxicos e com maior eficácia sob a doença.

O ozônio possui ação oxidativa e desinfectante e é efetivo contra os microrganismos, possui característica anti-inflamatória, analgésica e imunoestimulante. Apresenta baixo custo quando comparado com outros tratamentos, sendo de fácil aplicação e não possuindo efeitos colaterais e não gerando resíduos, pois se transforma em oxigênio e é reabsorvido (Freitas, 2011).

\section{Conclusão}

Com base nos resultados laboratoriais, na considerável melhora clínica e comportamental do paciente, concluiu-se, que neste caso, a ozonioterapia diminuiu os efeitos colaterais dos medicamentos, contribui na resposta imunológica e ajudou na cicatrização das lesões em pele.

\section{Referências bibliográficas}

Araújo, C. d. M. C., Costa, A. S. \& Risso, J. M. R. (2018). Uso da miltefosina como terapia combinada em leishmaniose visceral canina-relato de caso. Enciclopédia Biosfera, 15(27):106-116.

Bocci, V., Zanardi, I. \& Travagli, V. (2011). Oxygen/ozone as a medical gas mixture. A critical evaluation of the various methods clarifies positive and negative aspects. Medical Gas Research, 1(6):1-9.

Costa, C. H. N. (2011). How effective is dog culling in controlling zoonotic visceral leishmaniasis? A critical evaluation of the science, politics and ethics behind this public health policy. Revista da Sociedade Brasileira de Medicina Tropical, 44(2):232-242.

Freitas, A. I. A. (2011). Eficiência da ozonioterapia como protocolo de tratamento alternativo das diversas enfermidades na Medicina Veterinária. PUBVET, 5Art. 1192-1198.

Penido, B. R., Lima, C. A. \& Ferreira, L. F. L. (2010). Aplicações da ozonioterapia na clínica veterinária. PUBVET, 4Art. 974-979.

Schimming, B. C. \& Silva, J. R. C. P. (2012). Leishmaniose visceral canina - Revisão de literatura. Revista Científica Eletrônica de Medicina Veterinária, 10(18):1-5.

Silva, T. C. d., Shiosi, R. K. \& Raineri Neto, R. (2018). Ozonioterapia: um tratamento clínico em ascensão na medicina veterinária-revisão de literatura. Revista Científica de Medicina Veterinária, 15.

Sousa, C. M. H. S. \& Winck, C. A. (2018). Leishmaniose visceral canina: revisão de literatura. Revista da Universidade Vale do Rio Verde, 16(1):1-12.

Vilarindo, M. C., Andreazzi, M. A. \& Fernandes, V. S. (2013). Considerações sobre o uso 14 da ozonioterapia na clínica veterinária. Paper presented at the Resumos do VIII EPCC-Encontro Internacional de Produção Científica Cesumar, Maringá, Paraná, Brasil.

Recebido: 17 de setembro, 2019.

Aprovado: 31 de dezembro, 2019.

Publicado: 29 de fevereiro, 2020.

Licenciamento: Este artigo é publicado na modalidade Acesso Aberto sob a licença Creative Commons Atribuição 4.0 (CC-BY 4.0), a qual permite uso irrestrito, distribuição, reprodução em qualquer meio, desde que o autor e a fonte sejam devidamente creditados. 